\title{
Les avancées d'AMIMA sur les interactions océan-atmosphère
}

\section{Résumé}

Les résultats acquis depuis les campagnes AMMA/EGEE de 2005-2007 sur les interactions océan-atmosphère sont exposés. Ils montrent que la langue d'eau froide (LEF) dans l'Atlantique équatorial joue un rôle clé sur la mousson africaine. Le renforcement des alizés de sud-est, associés à l'anticyclone de Sainte-Hélène, favorise le pré-conditionnement de la thermocline et le développement de la LEF. De part et d'autre du front équatorial qui matérialise sa limite nord, les flux de surface très contrastés affectent la circulation dans la basse atmosphère. Cette circulation contribue à faire migrer la convection vers le nord et influence le saut de mousson.

\section{Abstract}

\section{Main results of AMMA on air-sea interactions}

The results gathered since the AMMA/EGEE campaigns of 20052007 in the field of air-sea interactions are presented. They show the key role of the Atlantic cold tongue (ACT) in the eastern equatorial Atlantic on the West African monsoon. The strengthening of southeasterlies, associated with the SaintHelena anticyclone, promotes the preconditioning of the thermocline along the equator and the development of the ACT. The equatorial front on its northern boundary, modifies the surface heat fluxes that affect the circulation in the lower atmosphere. This circulation helps to push moisture northward, toward the West African sub-continent, and impacts the monsoon jump.

\author{
Guy Caniaux ${ }^{(1)}$, Hervé Giordani ${ }^{(1)}$, Jean-Luc Redelsperger ${ }^{(2)}$, \\ Malick Wade ${ }^{(3)}$, Bernard Bourlès ${ }^{(4)}$, Denis Bourras ${ }^{(5)}$, \\ Gaëlle de Coëtlogon ${ }^{(6)}$, Yves du Penhoat ${ }^{(4)}$, Serge Janicot ${ }^{(7)}$, \\ Erica Key ${ }^{(8)}$, Urbain Koffi ${ }^{(9)}$, Nicolas Kolodziejczyk ${ }^{(2)}$, \\ Laurence Eymard( $^{(7)}$, Julien Jouanno ${ }^{(4,10)}$, Alban Lazar ${ }^{(3,7)}$, \\ Marion Leduc-Leballeur ${ }^{(6)}$, Nathalie Lefèvre ${ }^{(7)}$, Frédéric Marin $^{(4)}$, \\ Hanh Nguyen $^{(11)}$ et Gaëlle Parard(7) \\ (1) CNRM/GAME, Météo-France/CNRS, 42 avenue Gaspard Coriolis - \\ 31057 Toulouse \\ ( 2) LPO, CNRS-IFREMER-IRD-UBO, Plouzané, France \\ ( 3) LPAOSF, Dakar, Sénégal \\ (4) LEGOS-IRD-UPS, Toulouse, France \\ ( 5) LATMOS-IPSL, Vélizy-Villacoublay, France \\ (6) LATMOS-IPSL, Paris, France \\ ( 7) LOCEAN-IPSL, Paris, France \\ (8) National Science Foundation, Arlington, Virginia, États-Unis \\ ( 9) LAPA-MF, Université de Cocody, Abidjan, Côte d'Ivoire \\ (10) CICESE, Km 107 Carr, Tijuana-Ensenada, Ensenada, Mexique \\ (11) DEAS, University at Albany, SUNY, New York, États-Unis
}

a mousson ouest-africaine est une migration saisonnière des précipitations de l'équateur vers les régions du Sahel ; celles-ci débutent en mai sur la côte guinéenne, gagnent le Sahel en août et redescendent vers le golfe de Guinée en novembre. Sa mise en place se caractérise par un « saut», une apparente diminution des précipitations vers $7^{\circ} \mathrm{N}$. La connaissance de la période du saut de mousson est essentielle pour l'agriculture du Sahel de type pluviale, mais elle reste à ce jour incomplète.

L'une des ambitions du programme AMMA (Analyses multidisciplinaires de la mousson africaine) était d'améliorer notre compréhension de la mousson (Redelsperger et al., 2006). Les expériences de terrain de 2005-2007 ont été le point culminant du programme, en mobilisant des moyens humains et instrumentaux sans précédent, tant sur le continent que sur l'océan.

Sur l'océan, les campagnes EGEE (Étude de la circulation océanique et des échanges océan-atmosphère dans le

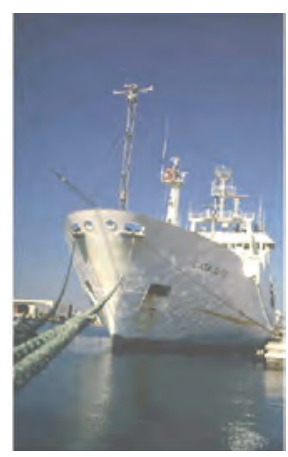

Le N/O L'Atalante équipé du mât instrumenté installé sur le pont avant pour la mesure des paramètres météorologiques et des flux turbulents Le tangon horizontal la proue est équipé pour la mesure du bilan radiatif de surface.

golfe de Guinée) ont débuté en 2005 avec, en 2006, le déploiement de trois navires océanographiques : N/O Ron Brown américain, Meteor allemand et L'Atalante de l'IFREMER (Bourlès et al., 2007), fait unique depuis les expériences du GARP en 1979.

Dans cet article, nous présentons les avancées réalisées dans le domaine des interactions océan-atmosphère depuis les campagnes EGEE. Elles ouvrent des perspectives intéressantes en termes de prévision du saut de mousson et de diagnostic pour les modèles couplés, qui peinent à reproduire la variabilité tropicale Atlantique. 


\section{La langue d'eau froide}

\section{Langue d'eau froide et vents sur l'Atlantique équatorial Est}

L'Atlantique équatorial Est est la région du bassin Atlantique tropical où le cycle saisonnier des températures de surface de l'océan (TSO) est le plus marqué. Elles chutent de 5 à $7{ }^{\circ} \mathrm{C}$ au printemps. Cette baisse apparaît vers $10^{\circ} \mathrm{W}$ et s'étend à la zone au sud de l'équateur comprise entre les côtes africaines et $20^{\circ} \mathrm{W}$. Elle s'amorce fin avril, s'accélère en juin et perdure jusque fin-juillet : c'est la langue d'eau froide (LEF, figure 1).

La LEF constitue une anomalie au refroidissement saisonnier des eaux de 1'hémisphère austral. Alors que la baisse des TSO devrait se propager du sud vers le nord, en suivant le déplacement saisonnier du soleil, la LEF anticipe de plusieurs mois le refroidissement sud-hémisphérique et affecte rapidement toute la bande sudéquatoriale. Ce n'est que vers septembre qu'elle se confond avec les eaux froides de l'hémisphère Sud.

Très tôt, les observateurs ont noté que cette chute de température coïncide avec le renforcement des alizés de sud-est. Entre mars et mai, l'anticyclone de Sainte-Hélène remonte vers le nord et les vents se renforcent au sud de l'équateur. Entre avril et juin, ce renforcement gagne le golfe de Guinée où les vents virent au sud-ouest après avoir franchi l'équateur.

\section{Phénoménologie 2005-2006: rôle du vent}

Les années 2005 et 2006 ont été très contrastées : en juin 2005, la LEF a été froide (figure $2 \mathrm{a}$ ) et les vents sur l'Atlantique équatorial soutenus, alors que la mousson était précoce et active. En juin 2006, les TSO furent chaudes (figure 2b), les vents faibles sur l'océan, la mousson tardive et peu intense (Janicot et al., 2008).

Marin et al. (2009) ont montré que le contraste de TSO en juin provenait d'une différence de phase spectaculaire de la LEF, ce qui s'est traduit, entre les mois de juin de ces deux années, par une différence persistante de $3^{\circ} \mathrm{C}$. Ce contraste a coïncidé avec un coup de vent soutenu à la mi-mai 2005 et une thermocline peu profonde en 2005. Le coup de vent précoce de mai a eu une répercussion immédiate, déclenchant subitement la baisse des TSO.

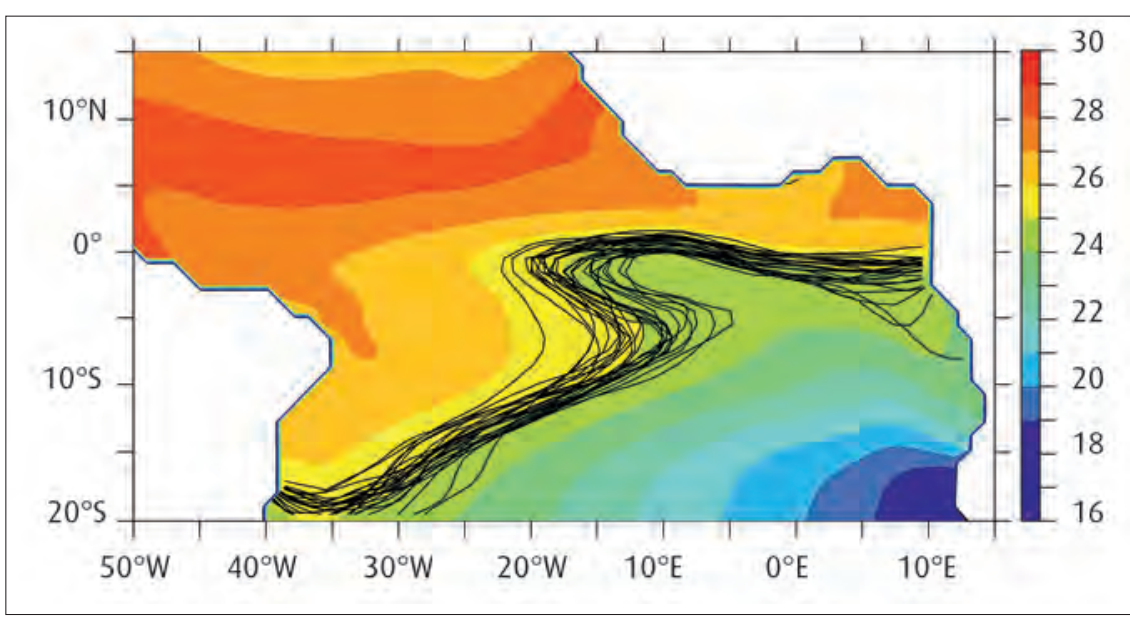

Figure 1- Position de l'isotherme $25^{\circ} \mathrm{C}$ délimitant la LEF lors des 30 dernières années (lignes noires). En couleur température de surface moyennes sur 30 ans (en ${ }^{\circ} \mathrm{C}$ ) lorsque la LEF atteint son extension moyenne annuelle. Sources : analyses Reynolds (d'après Caniaux et al., 2011).

Les différences 2005-2006 illustrent le lien étroit entre LEF et alizés. Elles ont permis de porter un regard nouveau sur la capacité du vent à refroidir localement l'océan équatorial, sur la variabilité

intra-saisonnière des vents de l'Atlantique équatorial Est dominée par une périodicité de 15 jours (de Coëtlogon et al., 2010 ; Janicot et al., 2011), sur le lien potentiel LEF-mousson ; enfin sur

Figure 2 - Cartes des températures de surface de l'océan les 15 juin 2005 (a) et 15 juin 2006 (b). À cette date, la LEF de 2005 est déjà bien formée avec un front équatorial net sur l'équateur, alors que les températures sont $3^{\circ} \mathrm{C}$ plus chaudes en 2006. Source : analyses Reynolds (d'après Marin et al., 2009).
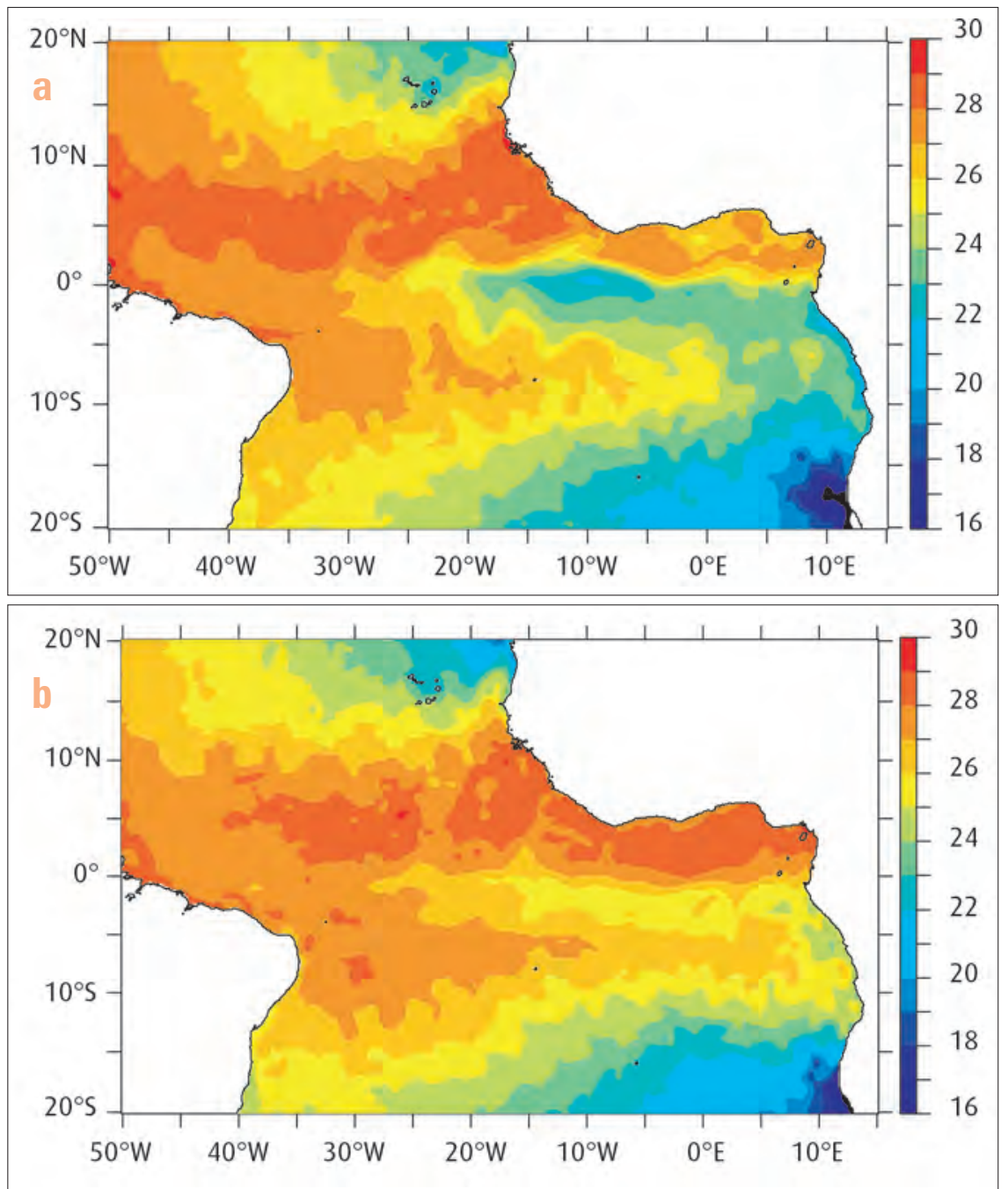
l'anticyclone de Sainte-Hélène, qui, à l'origine du renforcement des alizés, se trouvait mis dans la boucle des acteurs capables d'influencer la mousson.

\section{La théorie d'Ekman}

Quelle théorie permet d'interpréter le refroidissement de la LEF par renforcement des vents ? Plusieurs explications ont été proposées : advection horizontale d'eaux froides remontant le long des côtes africaines, advection verticale d'eaux froides sous-jacentes, remontée de la thermocline se propageant d'ouest en est par les ondes de Kelvin, mélange dû au cisaillement entre le courant équatorial sud et le sous-courant équatorial.

La théorie d'Ekman offre un cadre simple pour expliquer la réponse locale de l'océan au vent, car elle relie linéairement vitesse verticale et tension du vent de surface. Selon cette théorie, la vitesse verticale dans l'océan superficiel provient de quatre termes : la composante zonale, la composante méridienne, le rotationnel et la divergence de la tension du vent. Ainsi, la théorie d'Ekman appliquée aux vents du golfe de Guinée suggère une ascendance entre $3^{\circ} \mathrm{S}$ et l'équateur (synonyme de refroidissement) et une subsidence au nord de l'équateur. Cette configuration explique la forme de la LEF, confinée dans une étroite bande sud-équatoriale et une frontière nette à l'équateur (figure 1). En outre, les quatre termes contribuent tous à accroître les ascendances, d'où son expansion rapide en juin.

Cette théorie placerait la LEF au nord de l'équateur si des vents de nord-est soufflaient sur le golfe de Guinée au lieu d'alizés de sud-est. Autrement dit, ce sont la configuration géographique

Mise à l'eau d'une bouée dérivante Marisonde à bord du N/O L'Atalante lors de la campagne EGEE-3

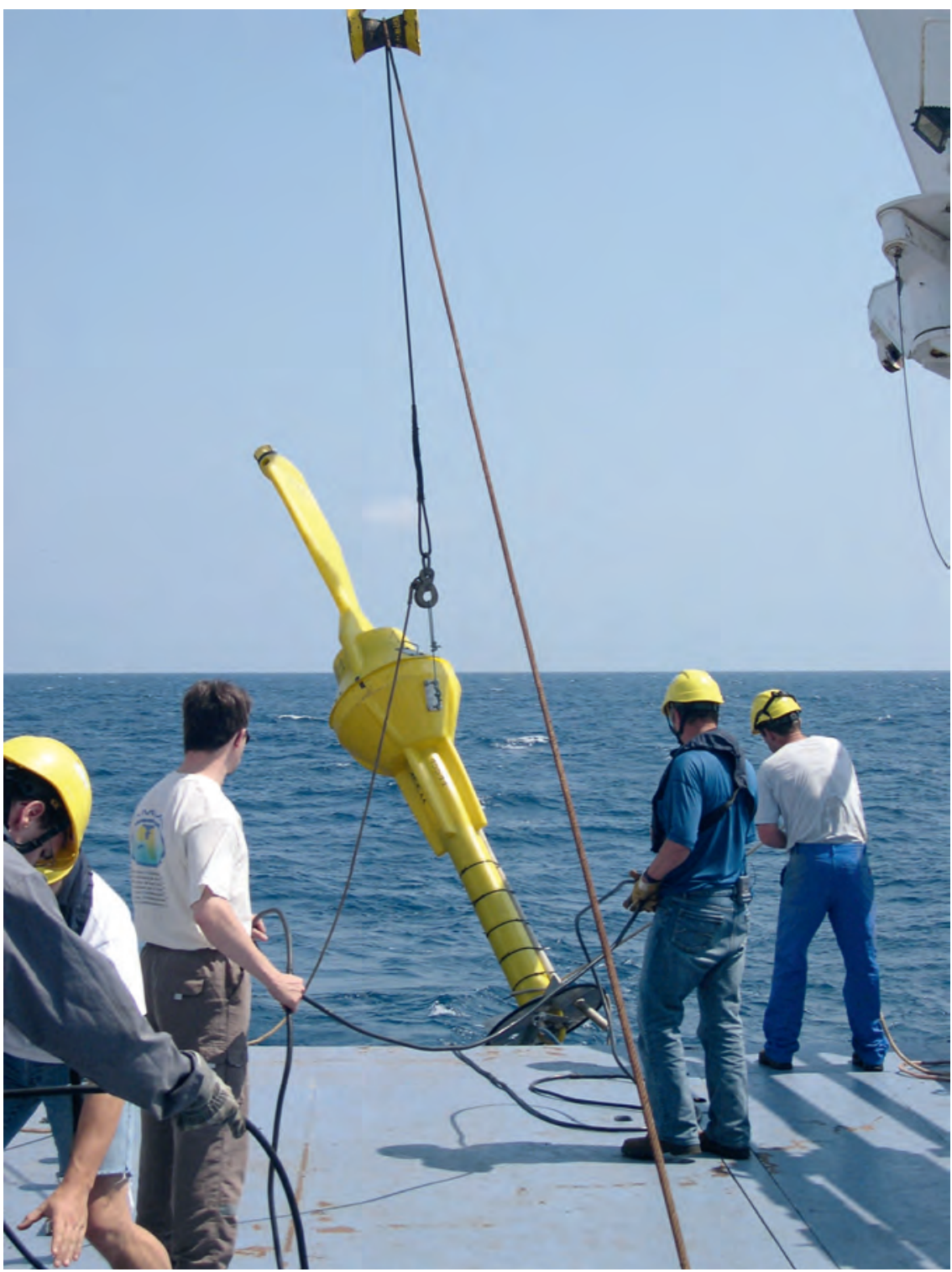

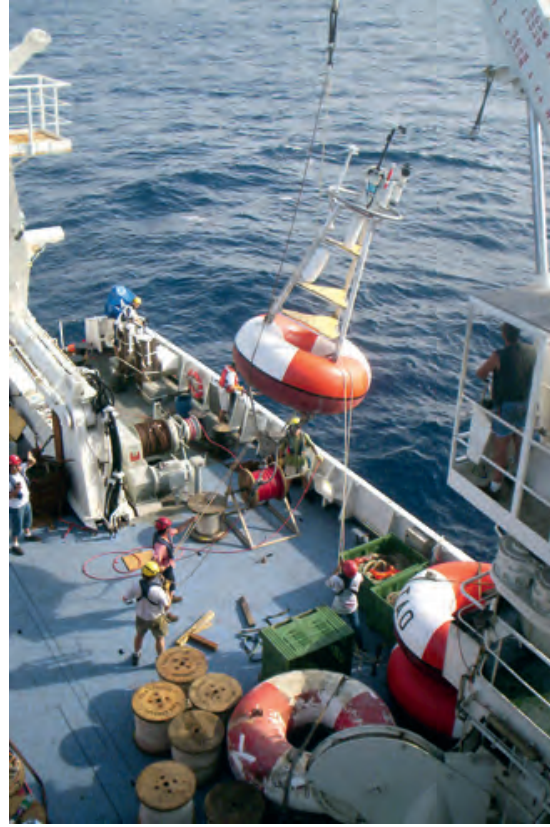

Activité sur le pont arrière du N/O L'Atalante pour la mise à l'eau d'une bouée ancrée du réseau PIRATA

du bassin et la circulation atmosphérique qui conditionnent l'emplacement de la LEF.

\section{Insuffisance}

\section{de la théorie d'Ekman}

Selon la théorie d'Ekman, les mouvements verticaux résultant de la divergence des courants engendrés par le vent à l'équateur, provoquent la remontée des eaux froides des profondeurs vers la surface. Ce processus est d'autant plus efficace que les profondeurs des couches mélangées sont faibles (d'où l'importance d'un préconditionnement lié à la position de la thermocline qui reflète un équilibre global). Cette vision a la faveur de nombreux auteurs, car elle établit un lien direct entre l'effet moteur du vent et le refroidissement de la LEF.

Or, dans les régions équatoriales, l'océan est très dynamique et non au repos comme le suppose la théorie. L'Atlantique équatorial se caractérise en effet par un système complexe de courants intenses, rapprochés en latitude (comme le courant de Guinée vers $3-4^{\circ} \mathrm{N}$ et le courant équatorial sud de sens contraire entre $4^{\circ} \mathrm{S}$ et $2^{\circ} \mathrm{N}$ ) et empilés sur la verticale, à l'image du courant équatorial sud surmontant le sous-courant équatorial dans les cent premiers mètres (Kolodziejczyk et al., 2009).

De plus, l'océan équatorial est stratifié et hétérogène, complexité supérieure aux hypothèses de la théorie. Les couches de mélange sont faibles à l'équateur $(10-20 \mathrm{~m})$ et s'approfondissent de 


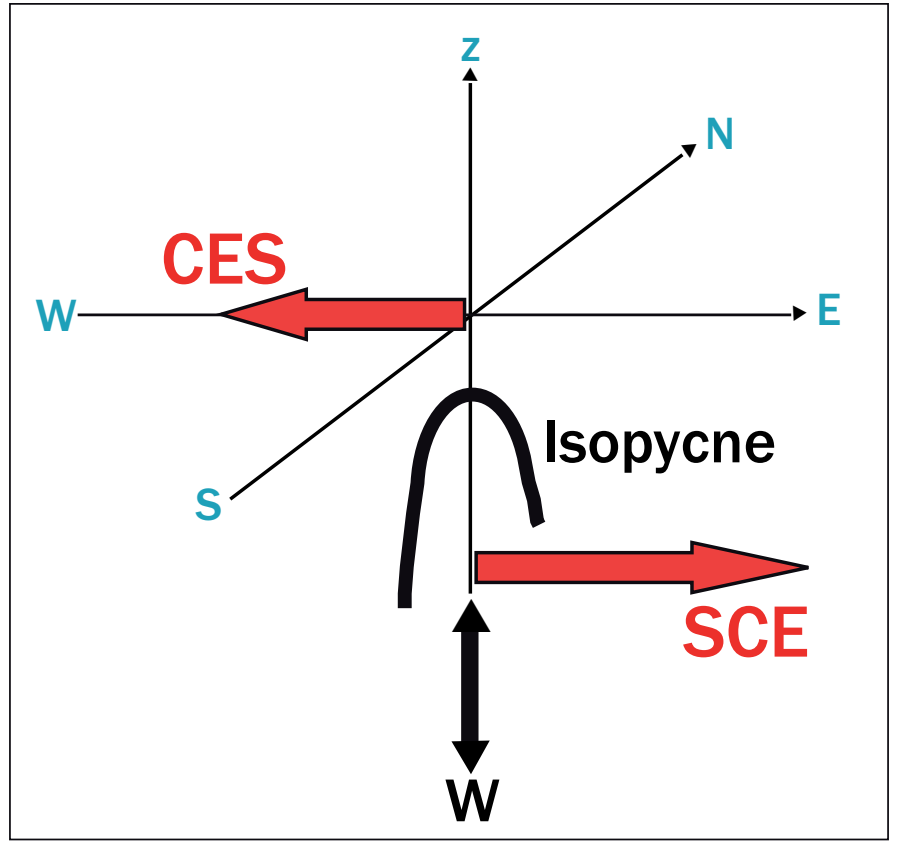

Figure 3 - Schéma de l'équilibre dynamique à l'équateur entre courants zonaux (flèches rouges) et vitesse verticale (flèche noire) La vitesse verticale W ajuste la concavité des isopycnes (courbe noire) au cisaillement vertical entre le courant équatorial sud (CES) en surface et e sous-courant équatorial (SCE) présent à partir de $30 \mathrm{~m}$ vers $10^{\circ} \mathrm{W}$ (d'après Giordani, 2011).

part et d'autre (d'environ $40 \mathrm{~m}$ vers $4^{\circ} \mathrm{S}$ ). Lorsque le vent souffle sur un océan hétérogène, les courants induits sont d'autant plus forts que les couches de mélange sont minces.

\section{Vitesses verticales et pré-conditionnement}

Le rôle des vitesses verticales comme éléments de pré-conditionnement de la LEF a été montré par Giordani et Caniaux (2011) à l'aide d'une simulation numérique. Celle-ci utilise les équations primitives et reproduit fidèlement les courants équatoriaux ainsi que l'hétérogénéité des couches de mélange. L'objectif était d'identifier les sources de vitesse verticale en utilisant la technique des oméga-équations (Pagé et al., 2007).

En tenant compte d'un niveau de complexité supérieure au système d'Ekman, l'équation de la vitesse verticale ne comporte plus 4 termes mais 18 ! Les résultats numériques montrent que la LEF se compose de deux upwellings : l'upwelling équatorial et celui du dôme d'Angola. Autre résultat majeur : l'existence d'un équilibre dynamique équatorial. Cet équilibre exprime la relation entre la courbure du champ de pression dans l'océan et une contrainte proportionnelle au cisaillement vertical du courant zonal. Lorsque l'équilibre est rompu, suite au renforcement des alizés, les vitesses verticales tendent à rétablir celui-ci en augmentant la courbure du champ de pression, d'où la remontée des isopycnes (lignes d'égale valeur de densité) vers la surface (figure 3). L'upwelling équatorial résulte du retour à l'équilibre dynamique que le vent détruit. Il apparaît comme une réponse indirecte au forçage du vent et non plus directe comme le suggérait la théorie d'Ekman.

Le rôle du vent sur un océan en mouvement apparait : l'accélération des alizés renforce le courant équatorial sud dirigé vers l'ouest. En présence du souscourant équatorial, qui porte dans la direction opposée, le cisaillement vertical du courant augmente et la thermocline remonte. Cette nouvelle théorie met en lumière le rôle fondamental de la vitesse verticale pour pré-conditionner l'océan et rapprocher la thermocline de la surface.

\section{Le refroidisse- ment dans la LEF}

\section{L'advection verticale}

La capacité des vitesses verticales à refroidir la LEF a été suggérée dès les années 1980. Malgré tout, les mouvements verticaux dans 1'Atlantique équatorial restent une thématique actuelle (exemples Lefèvre et al., 2008 ; Rhein et al., 2010), et ce d'autant plus que les données d'EGEE indiquent que l'Atlantique équatorial Est est une source importante de $\mathrm{CO}_{2}$ pour l'atmosphère (Lefèvre et al., 2009 ; Parard et al., 2010).

Or, Foltz et al. (2003) montrent à partir d'observations que le refroidissement dans la LEF induit par l'advection verticale est faible comparé aux autres processus. Ces résultats sont confirmés par des études numériques (Peter et al., 2006 ; Jouanno et al., 2011a) qui indiquent que l'impact de l'advection verticale sur le bilan de chaleur dans la couche mélangée est faible sur toute l'étendue et pendant toute la durée de la LEF. Si ce n'est pas l'advection verticale, il s'agit alors de savoir par quel mécanisme les eaux froides de subsurface sont apportées vers la surface.

\section{Cisaillement vertical et diffusion turbulente}

Grâce aux campagnes EGEE, un bilan de chaleur a été réalisé par Wade et al. (2011a) à l'aide de flotteurs PROVOR, dont le nombre a considérablement augmenté pendant les campagnes. Presque tous les termes de ce bilan (advections horizontale et verticale, flux air-mer, entraînement...) ont été évalués le long de la trajectoire de chaque flotteur. Les termes manquants (mélange vertical et diffusion horizontale) ont été traités comme résidu pour clore le bilan.

Lancement d'une radiosonde à partir de la passerelle du N/O L'Atalante pendant la campagne EGEE-3.

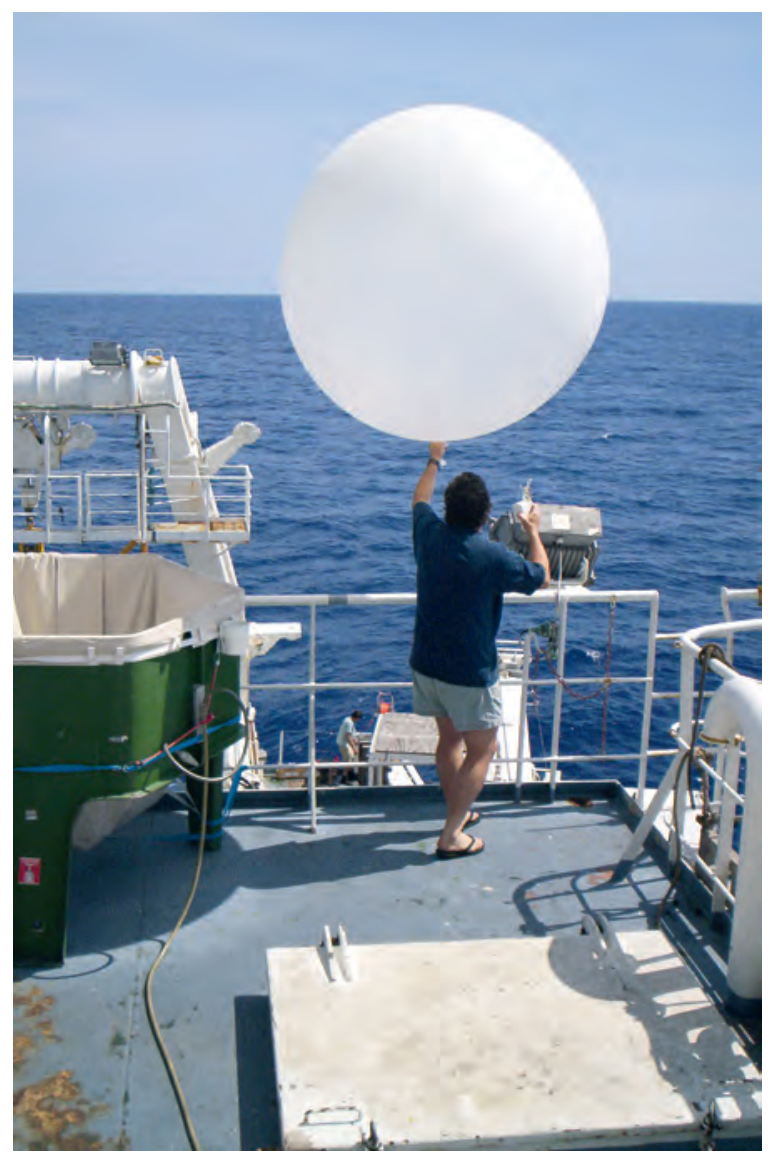


À l'échelle saisonnière, le résidu devient important dans la LEF, avec un cycle saisonnier marqué. L'évaluation des erreurs expérimentales permet d'attribuer une large part du résidu aux termes physiques non évaluables. Leur bonne correspondance avec des observations indépendantes de dissipation d'énergie cinétique turbulente, suggère que le mélange vertical est une source majeure du refroidissement dans la LEF. Ce mélange serait une conséquence du cisaillement vertical intense entre le courant équatorial sud et le sous-courant équatorial.

Ces résultats s'accordent avec ceux établis sur la base de simulations numériques (Peter et al., 2006 ; Jouanno et al., 2011b ; Wade et al., 2011b). À l'échelle saisonnière, ces résultats suggèrent un lien étroit entre l'accélération du courant équatorial sud et la formation de la LEF, par modulation du cisaillement vertical à la base de la couche mélangée. Les différences interannuelles montrent, par ailleurs, que la LEF précoce de 2005 proviendrait d'un mélange vertical accru en mai (Wade et al., 2011a).

\section{Régionalisation}

Le bilan de Wade et al. (2011a) a été régionalisé en découpant 1'Atlantique équatorial Est en sous-domaines de $9^{\circ}$ de longitude sur $6^{\circ}$ de latitude. Des évolutions très variables sont observées dans chaque zone, ce qui indique une forte régionalisation des processus, à laquelle s'ajoutent des différences saisonnières et interannuelles notables. C'est dans la LEF que le stockage thermique est le plus faible. De manière

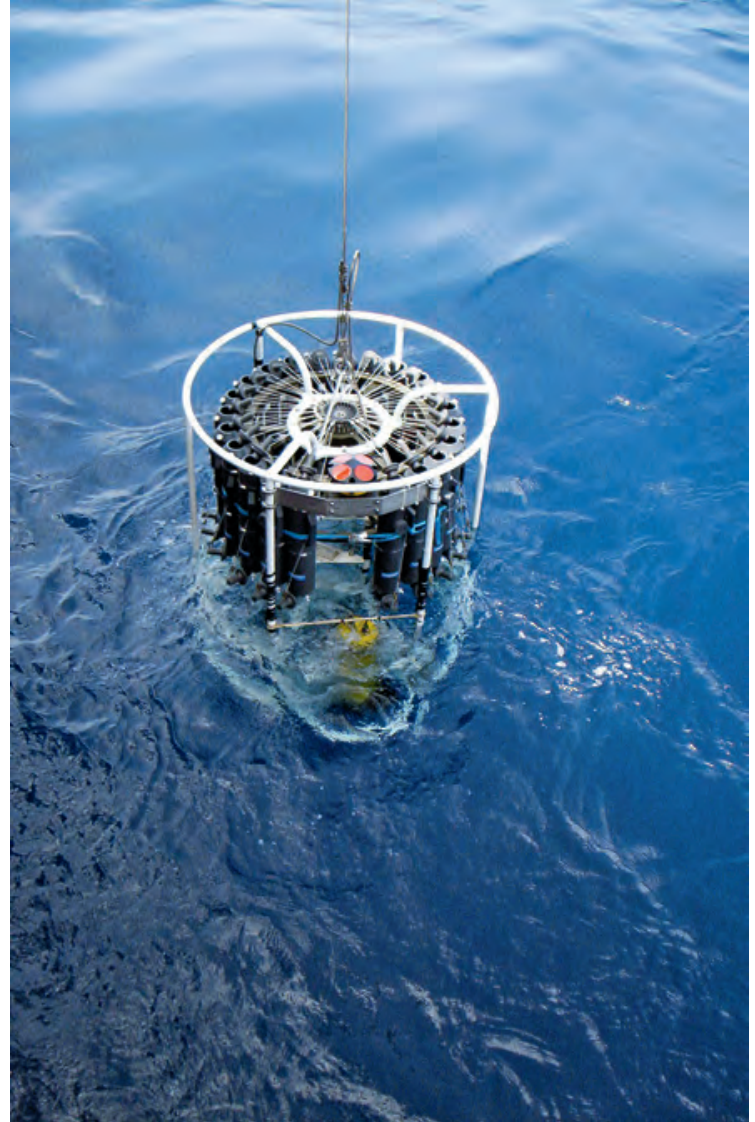

le terme de résidu est fort dans la LEF, il l'est aussi plus au nord, dans le golfe de Guinée. Cela suggère à nouveau que le cisaillement vertical est primordial dans cette région pendant la saison froide. Il trouverait son origine entre le courant de Guinée en surface, courant dirigé vers l'est et le courant de sub-surface portant vers l'ouest.

\section{Les gradients trans-équatoriaux de TSO}

Au nord de la LEF, les eaux chaudes $\left(30{ }^{\circ} \mathrm{C}\right.$ en avril) se refroidissent à la fin du printemps boréal. Mais ce phénomène débute en juin, un mois après la LEF, et il est plus lent. Par conséquent, le contraste de température entre les eaux chaudes au nord et froides au sud de

Mise à l'eau de la CTD (Conductivity-TemperatureDepth) pour mesurer températures, salinités, fluorescences et sels nutritifs dans l'océan.

étonnante, il reste négatif peu de temps pendant la saison froide, alors que le refroidissement saisonnier y est le plus intense. Or, le terme de stockage traduit l'évolution d'un contenu thermique, une température intégrée sur l'épaisseur de la couche de mélange. Ainsi, le paramètre le plus pertinent de la LEF n'est pas la température mais l'épaisseur de la couche de mélange. Autre résultat : si l'équateur s'accroît : ainsi naît le « front équatorial Atlantique », un front océanique saisonnier, dont le gradient nordsud mesuré en juillet 2006 lors d'EGEE-3 atteignait $4,5^{\circ} \mathrm{C}$ sur $100 \mathrm{~km}$ (figure 4 ; Lefèvre, 2009). Ce front est aussi marqué en salinité et en $\mathrm{CO}_{2}$

Figure 4 - Températures (en rouge, en ${ }^{\circ} \mathrm{C}$ ) et salinités (en bleu) enregistrées par le thermo-salinographe du N/O L'Atalante lors de la traversée du front équatorial Atlantique à $3^{\circ} \mathrm{E}$ dans le sens sud-nord, le 3 juillet 2006 (d'après Lefèvre, 2009).

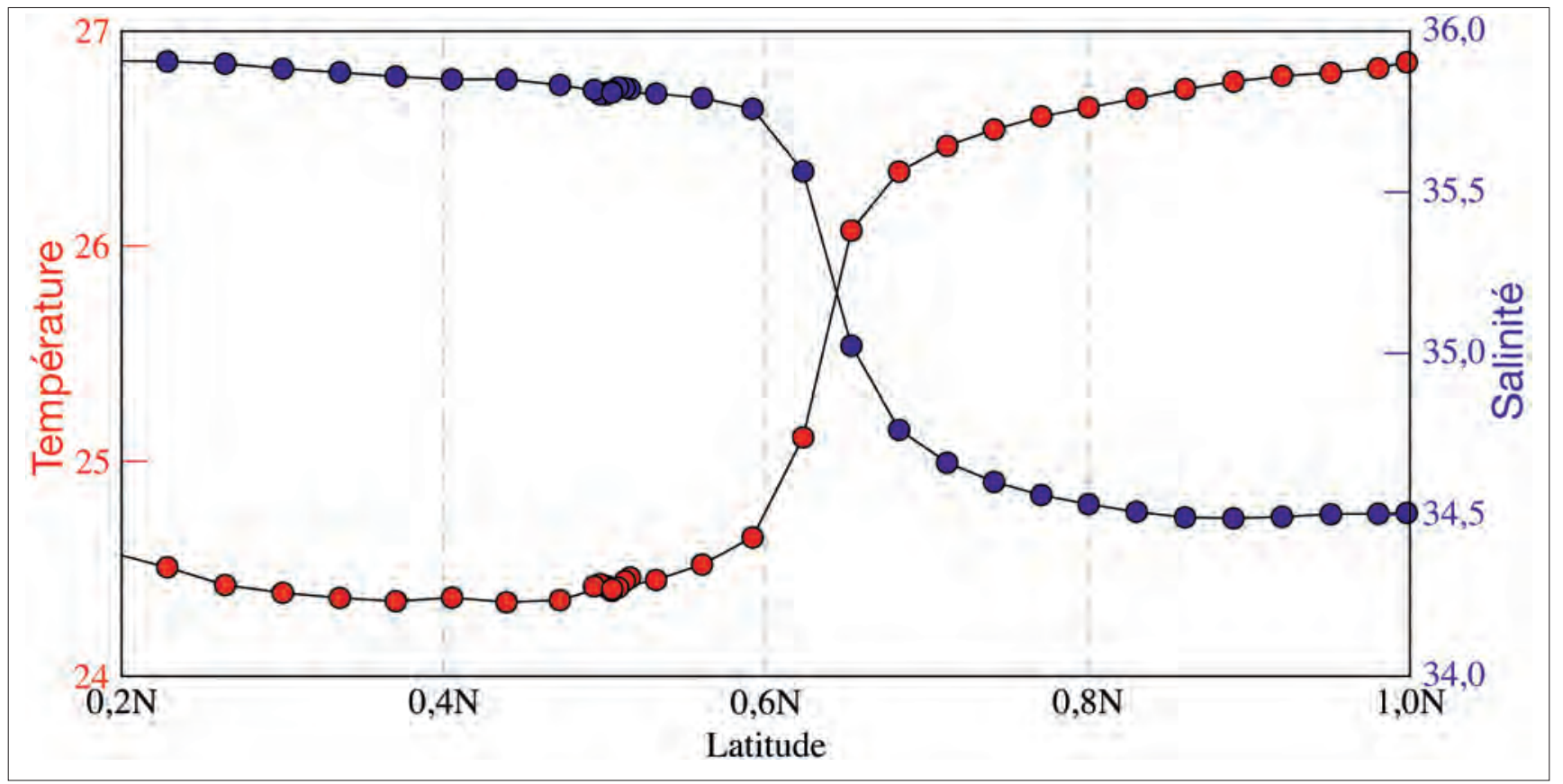


(Koffi et al., 2010). Les gradients proviennent des différences d'intensité des processus de part et d'autre de l'équateur. Wade et al. (2011a) montrent qu'il existe entre ces régions des différences de mélange vertical à la base de la couche de mélange, ainsi que des différences de flux de chaleur en surface. Cette configuration prouve l'existence de gradients trans-équatoriaux de divergence des flux turbulents, qui initient la frontogenèse océanique, laquelle pourrait être amplifiée par la configuration des courants.

En résumé, le renforcement des alizés de sud-est, lié à la dynamique de l'anticyclone de Sainte-Hélène, favorise la remontée de la thermocline, élément clé du pré-conditionnement océanique. En injectant de la quantité de mouvement à l'océan, le renforcement des alizés augmente le cisaillement vertical entre le courant équatorial sud et le souscourant équatorial. Le mélange vertical qui en résulte permet d'incorporer les eaux froides des profondeurs dans la couche de mélange : c'est la source majeure du refroidissement de la LEF. L'efficacité de ce mécanisme contraste avec ce qui se passe plus au nord, où le mélange vertical s'intensifie plus tardivement. Ces contrastes nord-sud se matérialisent par l'apparition du front équatorial.

\section{LEF et couplage avec la mousson}

\section{Variabilité interannuelle de la LEF}

Il existe une variabilité interannuelle de la LEF, qui s'est manifestée de manière remarquable entre 2005 et 2006, avec des coups de vents précoces en 2005 et tardifs en 2006 , situation à l'origine d'un déphasage spectaculaire de la LEF entre ces deux années. Pour caractériser cette variabilité, des indices permettant de signer les caractéristiques de la LEF ont été définis : indice de température, indice d'extension, dates d'apparition et de dissipation (Caniaux et al., 2011). Cette étude menée sur les 30 dernières années montre qu'il existe des variabilités interannuelles marquées de ces indices. Or, les années précoces pour la LEF, ne sont pas nécessairement des années froides. De même, une année tardive ne signifie pas une année chaude ou une LEF peu étendue. Autrement dit, il n'existe pas de corrélations fortes entre ces indices, ce qui

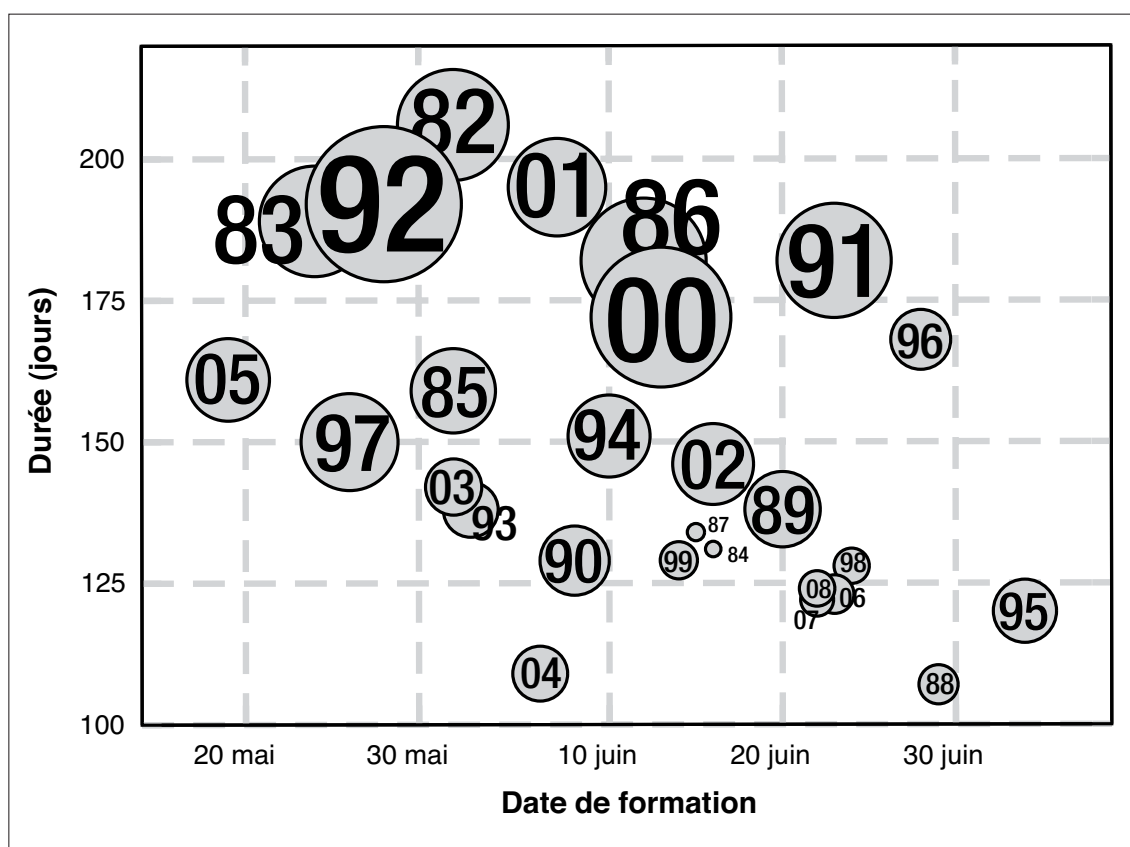

Figure 5 - Diagramme durée (en ordonnée) - date de formation (en abscisse) - extension de la langue d'eau froide (grosseur des cercles ; plus la LEF est étendue, plus le cercle est large) pour les années comprises entre 1988 et 2007 (d'après Caniaux et al., 2011).

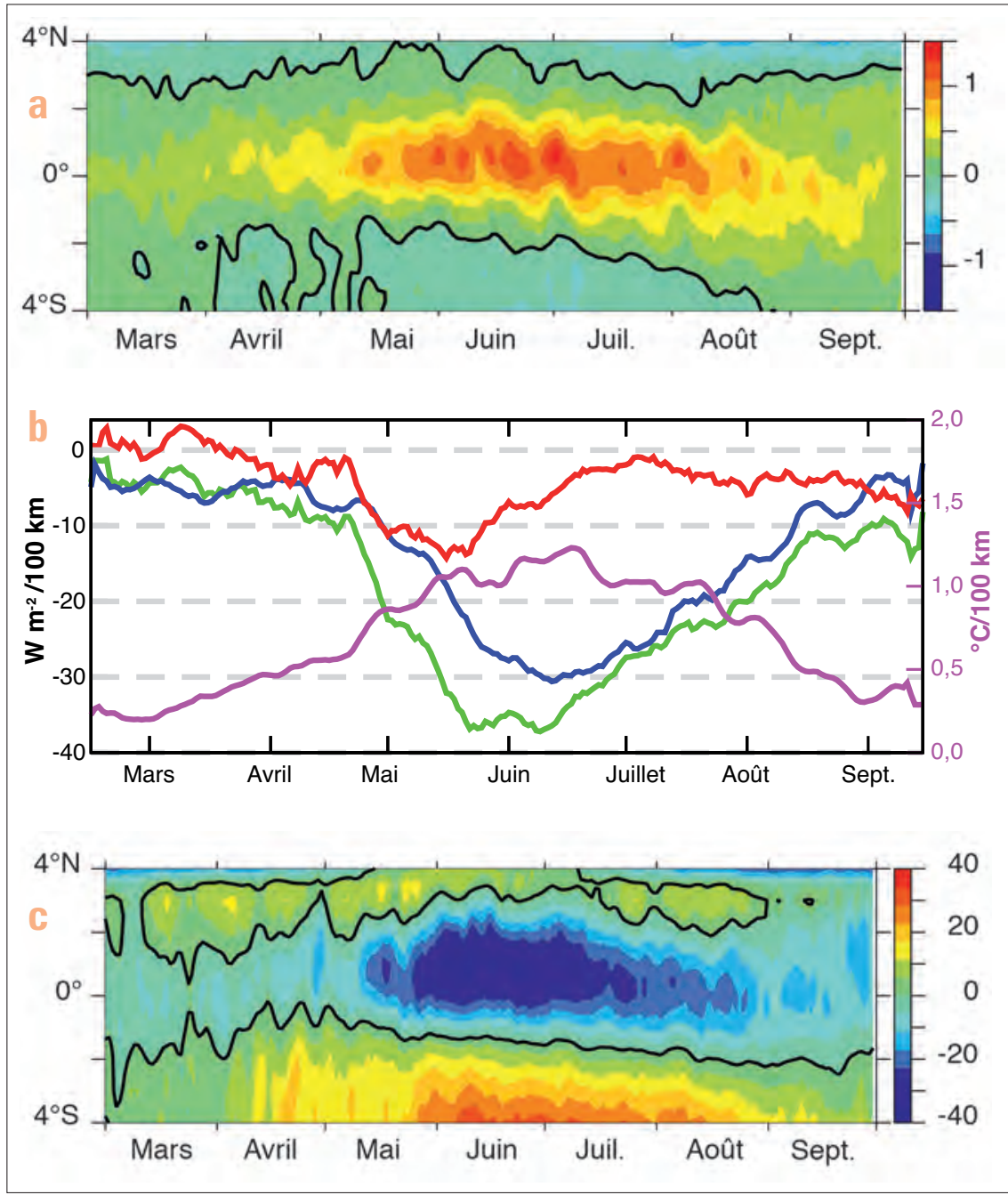

Figure 6 - Diagramme latitude-temps des gradients méridiens de : (a) TSO et (c) flux net. Évolution temporelle des gradients méridiens à $0,5^{\circ} \mathrm{N}$ de : (b) TSO (magenta), flux solaire (rouge), flux non-solaire (bleu) et flux net (vert). Les gradients de TSO sont en ${ }^{\circ} \mathrm{C} / 100 \mathrm{~km}$ et ceux de flux en W m²/100 km ; ils sont moyennés entre 1998 et 2007 et entre $10^{\circ} \mathrm{W}$ et $4^{\circ} \mathrm{E}$. Sources : analyses Reynolds et CEPMMT (d'après Caniaux et al., 2011). 
suggère des sources multiples de variabilité (figure 5). Un mode de variabilité a été identifié par Marin et al. (2009) : des coups de vents intenses et précoces déclenchent des LEF précoces, comme en 1997 et en 2005. Hormann et Brandt (2007) montrent que le renforcement du sous-courant équatorial serait corrélé à l'intensité du refroidissement de la LEF. La théorie de l'équilibre dynamique équatorial permet d'expliquer ce second mode : l'intensification du cisaillement vertical entre un sous-courant plus fort et les courants de surface favorise une remontée de la thermocline et un mélange vertical plus intense, d'où une LEF plus froide (figure 3). Brandt et al. (2011a) montrent que la dynamique des courants équatoriaux profonds pourrait contribuer à la variabilité de la LEF à l'échelle interannuelle. Malgré tout, d'autres facteurs de variabilité restent à évaluer.

\section{Gradients trans-équatoriaux de flux}

Les gradients méridiens de TSO dans le front équatorial atteignent leur intensité maximale en juin, entre $1^{\prime}$ équateur et $1^{\circ} \mathrm{N}$ (figures $6 \mathrm{a}, 6 \mathrm{~b}$ ), pour glisser ensuite légèrement plus au sud. Ces différences nord-sud engendrent des différences de flux en surface qui modifient le flux de mousson. En région tropicale, le bilan des flux de surface est dominé par le flux onde courte (qui réchauffe l'océan) et par le flux de chaleur latente (qui le refroidit). Dans la LEF, avec ses basses TSO, les flux de chaleur latente sont faibles. Au nord, à l'inverse, les TSO étant plus chaudes, ils sont plus fortement négatifs : d'où l'apparition de gradients méridiens marqués, comme le montrent les flux recueillis lors d'EGEE-3 (Bourras et al., 2009). En mai-juin, le flux onde courte présente également un gradient méridien (figure 6b). Ce gradient s'explique par la différence de nébulosité entre le nord et le sud de l'équateur, alors que les masses nuageuses de la zone de convergence intertropicale (ZCIT) recouvrent le golfe de Guinée. Ainsi, les gradients méridiens de flux net, dont la composante non-solaire reflète les gradients de TSO du front équatorial, sont amplifiés par ceux du flux onde courte. Négatifs de $2^{\circ} \mathrm{S}$ à $3^{\circ} \mathrm{N}$ entre avril et septembre, leurs extrêmes sont atteints plus tôt ( 25 jours) que ceux de TSO (figures $6 \mathrm{~b}$ et $6 \mathrm{c}$ ). Période primordiale, car ces contrastes accélèrent la circulation atmosphérique dans le golfe de Guinée.

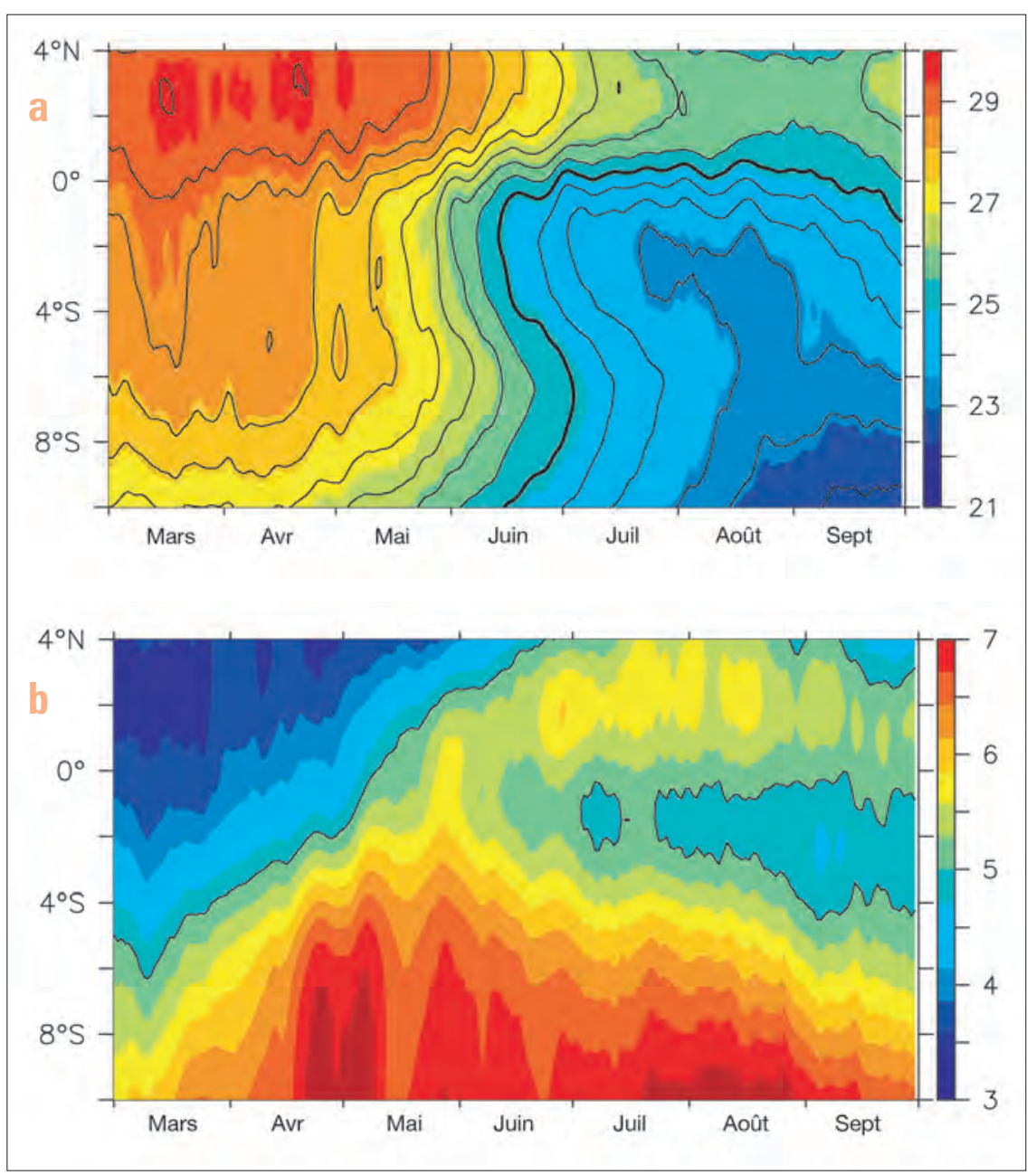

Figure 7 - Diagrammes latitude-temps : (a) des TSO $\left({ }^{\circ} \mathrm{C}\right)$ et $(\mathrm{b})$ des vitesses du vent de surface $\left(\mathrm{m} \mathrm{s}^{-1}\right)$. Les champs sont moyennés entre 1998 et 2007 et entre $10^{\circ} \mathrm{W}$ et $4^{\circ} \mathrm{E}$. Source : analyses Reynolds et CEPMMT (d'après Caniaux et al., 2011).

\section{Circulation atmosphérique trans-équatoriale}

Plusieurs études ont été consacrées aux circulations atmosphériques induites par des fronts océaniques. Ces travaux montrent qu'en affectant les vents, un front océanique modifie la stabilité, les gradients de pression et la distribution de l'humidité dans la couche limite atmosphérique. L'un des effets signant la présence du front équatorial est la décélération des vents sur les eaux de la LEF et leur accélération au nord. Ces perturbations ont été mises en évidence par de Coëtlogon et al. (2010), Brandt et al. (2011b), ainsi que par Caniaux et al. (2011) : lorsque les gradients de flux de surface se renforcent, les vents diminuent entre $2^{\circ} \mathrm{S}$ et l'équateur. Simultanément, les vents augmentent dans l'hémisphère Nord où leur intensité maximale est atteinte entre mi-juin et fin-août (figure 7). Or, le maximum de vent reste cantonné entre $1^{\circ} \mathrm{N}$ et $3^{\circ} \mathrm{N}$ : ce n'est donc pas la circulation atmosphérique sur l'Afrique de l'Ouest qui s'étend en direction de l'océan.
Autrement dit, le flux de mousson ne s'apparente pas à une brise de mer qui serait pilotée par la dépression thermique saharienne. Au contraire, sur l'océan, c'est la LEF qui modifie la circulation atmosphérique. Il existe donc un couplage entre l'océan et l'atmosphère. Dans un premier temps, l'anticyclone de Sainte-Hélène est responsable de la mise en place de la LEF, qui joue un rôle passif à ce stade. Peu de temps après, elle devient active car dès que les flux de surface sont suffisamment contrastés de part et d'autre du front équatorial, les vents accélèrent le flux de mousson.

\section{Front équatorial et précipitations côtières}

Les effets de la LEF sur la couche limite atmosphérique ont été examinés par Leduc-Leballeur et al. (2011). En utilisant les données d'EGEE-3, ces auteurs montrent que chaque coup de vent sur 1'Atlantique équatorial provoque un refroidissement de la LEF et une accélération des vents dans le golfe de 
Guinée, et ce sur des échelles de temps de 10 jours. Du sud au nord du front équatorial, la couche limite s'épaissit considérablement (figure 8). Une circulation locale se met en place, qui se superpose à la circulation de grande échelle, avec des vents faiblissant sur les eaux froides et se renforçant audessus des eaux chaudes. L'accélération des vents sur les eaux chaudes affecte la répartition de l'humidité, contribue à repousser la convection atmosphérique plus au nord et renforce les précipitations sur les côtes africaines (Leduc-Leballeur, 2012). Thorncroft et al. (2011) et Nguyen et al. (2011) se sont intéressés au déplacement des masses nuageuses précipitantes et définissent la " phase côtière de la mousson ». Sa mise en place suivrait de 10 jours celle de la LEF et précéderait celle du saut de mousson. De plus, sa variabilité serait étroitement associée à celle de la LEF. Contrairement à ce que l'on pensait au début du programme AMMA, l'influence de la LEF serait plus importante que celle de la dépression saharienne, non seulement pour la circulation au-dessus de l'océan, mais aussi pour la phase côtière de la mousson. Enfin, les dates d'apparition de la LEF, des pluies côtières et du saut de mousson seraient corrélées à plus de $60 \%$ (Caniaux et al., 2011 ; Nguyen et al., 2011). Ces chiffres suggèrent que si l'on parvient à identifier les facteurs de variabilité de la LEF, alors il existe un potentiel certain pour prévoir la phase côtière et le saut de mousson.

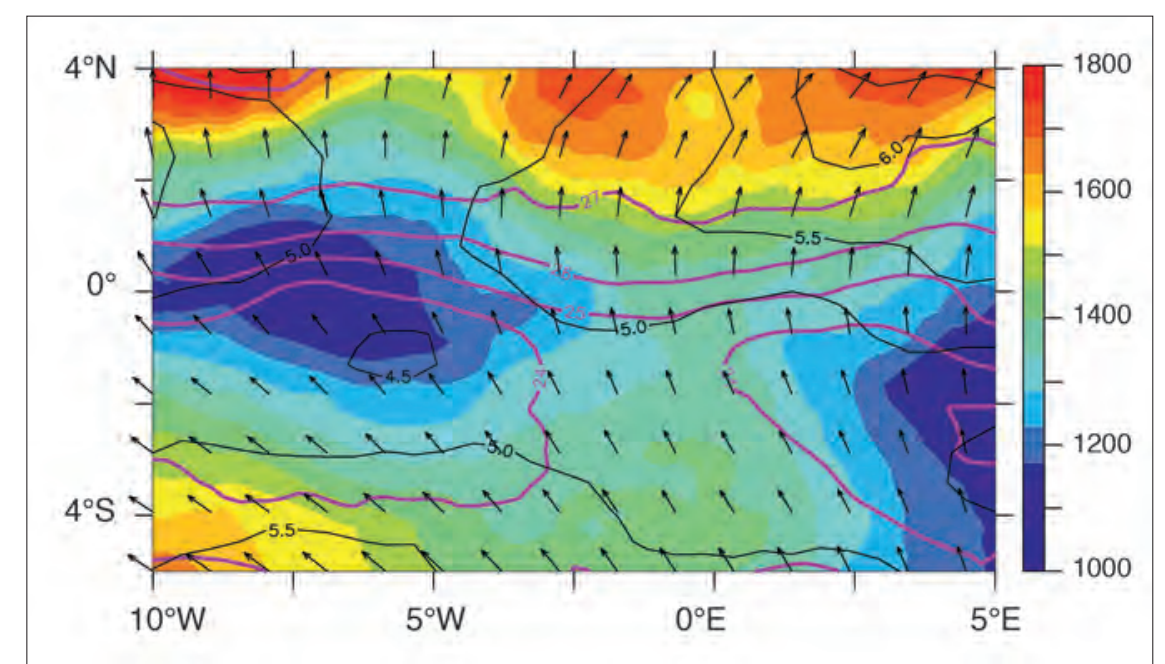

Figure 8 - Hauteur de la couche limite atmosphérique (en m) entre le $1^{\text {er }}$ et le 10 juillet 2006, TSO (isolignes magentas en ${ }^{\circ} \mathrm{C}$ ) et vents (flèches et isolignes noires en $\mathrm{m} \mathrm{s}^{-1}$ ). Sources : analyses CEPMMT et TMI (d'après Leduc-Leballeur et al., 2011).

\section{Conclusions}

Les expériences AMMA/EGEE ont fait considérablement progresser les idées sur le couplage océan-atmosphère au sud de l'Afrique de l'Ouest. Elles ont permis d'identifier les acteurs du couplage :

- l'anticyclone de Sainte-Hélène avec l'intensification des alizés ;

- la langue d'eau froide (LEF) avec ses faibles profondeurs de couche de mélange et le cisaillement vertical des courants horizontaux ;

- le front équatorial Atlantique et la circulation atmosphérique locale associée.
La LEF joue un rôle actif sur la mousson ouest-africaine. Cette phase a lieu lorsque les gradients de température sur le bord septentrional de la LEF s'intensifient. Ils engendrent des gradients de flux de surface, renforcés par des gradients de flux solaire résultant de différences nord-sud de nébulosité. À leur tour, les gradients de flux de surface deviennent le moteur d'une circulation locale qui se superpose à la circulation de grande échelle et qui contribue à faire migrer la convection atmosphérique vers le nord. Si l'on se donne les moyens d'identifier toutes ses sources de variabilité, la LEF renferme, par son influence sur la phase côtière et le saut de mousson, un potentiel prédictif pour les différentes phases de la mousson. 